Henry $A-$, aged twenty-five. Injury, without fracture, to the hip. Knocked down on the pavement by a cab.

Edward $\mathrm{M}-$, aged sixty-seven. Fracture of the tibia and fibula ; severe bruising of the integument, from a cab having knocked him down. The horse trampled on him.

John $\mathrm{E}$ ture of the os calcis, with laceration of the integuments of the heel, and complete severance of the tendo-Achillis, caused by a cab-wheel passing over it. In this case the wound has sloughed, the patient was delirious, but is now likely to recover.

$$
\text { Cart Accidents. }
$$

Wm. L-a aged fourteen. Knocked down by a cart. Bruise of the right arm.

Alfred $\mathrm{D}-\mathrm{C}$, aged nine. Fracture of the right femur through the lower third, caused by a cart-wheel passing over it. All the patients are convalescent.

The injuries received by women differ in some important respects from those received by men. An analysis of twenty cases yields the following results :- -

Harriet $\mathrm{H}-$, aged twenty-four, was stabbed in the chest by a man with whom she was quarrelling. The exact cause of the disturbance could not be ascertained. Many persons were involved, and the quarrel continued many hours; it originated in jealousy.

Eliza C-, aged twenty-four. Fracture of the jaw from a blow, supposed to have been given by her husband.

Louisa C-_ a a - a thirty-seven. Compound fracture of both thighs, and fracture of the left great toe; compound fracture of both arms; severe cut of head and eyebrow. She sprang from the parapet of a house in a fit of mania. Death.

Anne $J$ - aged fifty-five. Fracture of the os calcis, with separation of the fragments, from slipping off a step.

Mary W-, aged seventy-one. Severe cut-throat, self-inflicted; insanity; death.

Injuries received in parturition. To be removed to another ward.

Mary D - aged forty. Vesico-vaginal fistula, admitting three fingers; prolapsus of the bladder; obliteration of the urethra.

Mary R_-, aged twenty-two. Supposed fistulous passage between the vagina and rectum, proceeding from her first confinement two years and a half ago. Examination at present impracticable, on account of ulceration of the external organs.

\section{Burns and scalds.}

Rose $\mathrm{M}-$ aged thirteen. Burns of legs and arms from falling asleep over the fire.

Charlotte $\mathrm{H}-$, aged thirty. Extensive burn of arm and chest, caused by her falling into the fire in a fit.

Thomas W- aged ten months. Burn on both hands while being carried by the father from a house on fire.

Mary $\mathrm{N}$, aged seven. Extensive burn over the whole body; clothes caught fire; death.

Injuries received in the streets (three in number).

Alice $\mathrm{M}^{6} \mathrm{G}$ - aged fifty. Bruise of lower extremities. Knocked down by a van.

Mary K- aged sixty-seven. Lacerated wound of the arm. Knocked down by a van.

Anne $\mathrm{P}$ - , aged twenty-three. Bruise of the ankle Knocked down by a cab. In the fall she received a severe cut in the nates from the steel of her crinoline.

\section{Injuries received within doors.}

Ronzo Z-, an Italian boy. Fracture of the thigh from trying to walk alone in his father's shop.

Anne M-, aged forty-six. Cut head from fall down-stairs. Charlotte W Catherine M-_, aged eighty-two. Cut head from fall down-stairs.

Alice G- - aged thirty. Simple fracture of the tibia and fibula, from a kick, supposed by her husband.

Georgina $\mathrm{H}-$ - aged seven. Bruised elbow, from a fall. Queen Anne-street, Cavendish-square, April, 1866.

AFTER all the maladies which have attacked useful animals, the noxious animals now suffer in their turn. The reptiles confined in the Jardin des Plantes have, some of them, been carried off by disease, and a post-mortem examination has revealed the presence of worms in the lungs. Other reptiles have been attacked by typhus and have died.-Land and Water.
REMARKS

ON THB

\section{SYMPATHY BETWEEN THE AUDITORY CANAL AND THE LARYNX.}

\section{By CORNELIUS B. FOX, M.D. EurN., M.R.C.P. LoND.}

THE study of the sympathetic sensations and actions of the human body is one of great interest, not only on account of their number and variety, but in consequence of the obscurity with which so many of them are surrounded. The great importance of a thorough knowledge of this somewhat intricate subject to every medical man is so obvious as to require no demonstration. The sympathy between the ear and distant organs, such as the larynx, liver, uterus, spleen, \&c., is adverted to in many old medical works ; but the very general nature of the statements made, and the want of accurate investigations into these supposed connexions, oblige us either to accept the assertions cum grano salis, or seek to discover the amount of truth involved in them. The sympathy existing betwixt the ear and the larynx in some individuals, although well known to the older writers, would seem to have been overlooked by modern authors. One of the problems of Cassius Medicus was the following: "Why does irritating the earsas, for example, with a speculum-cause sometimes a congh, just as if the trachea was irritated?" Whytt, in his work on the Sympathy of the Nerves, published in 1767, refers to it, and states that when the trachea has been rendered more sensitive than usual by a catarrh, cough is more readily produced. by irritation of the auditory canal. His explanation of this, as well as of other sympathies, need not be referred to, on account of the well-known erroneous nature of his views respecting the influence of the mind on vital phenomena. Coming down to more recent times, we find that Kramer, in his work on Diseases of the Ear, published in 1837, makes the following solitary observation: "Tickling and scratching the meatus excite in the larynx a troublesome irritation to cough." Romberg states that "pruritus of the external meatus auditorius, from hyperæsthesia of the auricular branch of the vagus, is sometimes observed, and is accompanied with cough and vomiting." * The only references to this subject with which I am acquainted, in the principal recent works on medicine, are the following. Dr. C. J. B. Williams, in his Principles of Medicine, whilst enumerating the reflected and sympathetic sensations, writes: "Touching the external auditory meatus causes a tickling sensation of the epiglottis." Toynbee, in his work on Diseases of the Ear, says : "In certain cases, the presence of a foreign body in the meatus gives rise to coughing, and even to vomiting--symptoms which seem traceable to irritation of the auricular branch of the vagus nerve." Yearsley refers in very general terms to the alterations of the voice, as regards its pitch and quality, which accur in cases of deafness dependent on diswases of the ear, but does not otherwise allude to this connexion. Turning from the aurists to the laryngoscopists, we meet with no better success, so finally resort to a work on Cough, $\uparrow$ in which, amongst the occasional causes of this symptom, are to be found dyspepsia, giving rise to " stomach cough," hysteria, affections of the tonsils and uvula, uterine derangements, teething, worms in the intestines, organic and functional affections of the heart, disease of the liver, \&c. Although irritation of the auditory canal is not referred to as a cause, yet we know that cough is occasionally idiopatlic, which means in reality that we are sometimes entirely unable to discover the cause concerned in its production.

$$
\text { "Felix qui potuit rerum cognoscere causas." }
$$

Let us, then, endeavour to assist in the determination of every "fons et origo mali," that so we may as soon as possible expunge from our vocabulary the term "idiopathic." In my Graduation Thesis, "Concerning the Larynguscope and some Laryngeal Diseases" (June, 1864), presented to the University of Edinburgh, reference was made to the sympathy subsisting between the external ear and the larynx; and an explanation of this interesting connexion was advanced. The advisability of a careful examination of the auditory canal was also urged

* Vide a review of his Lehrbuch des Nervenkrar kheiten des Menschen in the British and Foreign Medical Review, April, 1814. + Semple on Cough, its Causes, Varieties, and Treatment. 
in all cases where an obstinate cough, whether laryngeal in character or otherwise, obtrudes itself as a prominent symptom. Further observation in this country and on the Continent has convinced me that a. state of hyperæsthesia of the nerve supplying the external auditory meatus is of frequent occurrence, and that a cough solely dependent on the existence of some irritation in that canal is by no means rare. I am well acquainted with a healthy gentleman, of nervous temperament, who has been annoyed, as long as he can remember, with a feeling of irritation in the larynx whenever he has introduced an earpick for a short distance within his left auditory canal. This sensation excites a violent cough of a suffocating or convulsive character. The organs of hearing appear on examination to be perfectly healthy, the sense being somewhat more acute on the affected side than on the other. A careful examination of his larynx by means of the laryngoscope assured me of the absence of any abnormality whatsoever. He finds that the excessive use of his voice, as in long-continued singing, produces a pain in the ear, which extends into the zygomatic fossa, and along the lower jaw towards the chin. Notes of a case are in my possession, too copious, however, to be transferred to this paper, in which a cough was solely produced by an irritation of the auditory canal in a healthy-looking woman of fifty years, who had spent much and profited nothing in her attempts to remove it. She had suffered from cough for eighteen months. The voice was unaltered, the cough was laryngeal in character, and most distressing. The throat, lungs, and other viscera were healthy. The larynx was shown by the aid of the laryngeal mirror to be normal, with the exception of a slight exaggeration of the usual rose tint of the parts. She complained of deafness in the right ear, the symptoms being those of an accumalation of cerumen. On examining the auditory canal, a large plug of hardened wax was found, and removed by means of injections. The appearance of a small amount of purulent discharge led to a further examinafion, resulting in the discovery of a small oval ulcer on the floor of the canal about one-eighth of an inch from the tympanic membrane. Almost immediate relief of the cough followed the extraction of the wax. After two or three applications of a solution of nitrate of silver to the ulcer, cicatrization was complete, and the woman was not only entirely free of the aural affection, but of the troublesome cough occasioned by it. Toynbee* refers to a patient under his care who suffered from a most intractable cough. He had a portion of dead bone in his external auditory meatus, which was removed. The withdrawal of this source of irritation was attended by an immediate disappearance of the cough, which no remedies had been able to subdue.

The sympathy between the ear and the larynx is manifested only in certain individuals, as has been already intimated. With the object of ascertaining the proportion of those subject to this peculiarity, eighty-six men and women have been recently examined by myself and others under my direction. This sympathetic connexion was found to exist in fifteen persons, which is equivalent to about seventeen per cent. There was only one in whom irritation of the auditory canal produced vomiting, and in this case some doubt was attached to the statement.

Two of the extracts above given refer to the nerve of the ear concerned in the production of this interesting sympathy. Romberg, whose opinion is endorsed by that of Mr. Toynbee, states that the auricular branch of the vagus is the nerve in volved. This view is most undoubtedly erroneous, for this branch of the vagus is not distributed to the auditory canal, as they seem to imagine, but to the posterior part of the pinna. The external auditory meatus is supplied with nerves from quite a different source-viz., from the auriculo-temporal branch of the inferior maxillary division of the fifth cranial nerve, whilst the tympanic cavity and its contents are furnished with nerves from this same fifth nerve, the glossopharyngeal and facial trunks, through the medium of the tympanic plexus and otic ganglion. If in those cases where an increased sensibility of the auditory canal is present during life, a prolongation into that tube of the tympanic branch (Jacobson's nerve) of the glosso-pharyngeal could be demonstrated after death, the intimate connexions of this nerve with the vagus would most readily account for the sympathy betwixt the auditory canal and the larynx. Kölliker tells us that the terminations of the tympanic nerves are unknown, and alludes in his " Microscopic Anatomy" to a statement made by Sappey to this effect-that the chief nerve of the membrana tympani is derived from the vagus, and lies in its outer layer of cutis. My own dissections have not tended to confirm the opinion of this anatomist. The nerves concerned, then, in this sympathetic connexion between the auditory canal and the larynx are without doubt, the branches of the auriculo-temporal of the fifth cranial nerve on the one hand, and the vagus, from which source the larynx is alone supplied, on the other. It is well known that an irritation of the peripheral extremity of one branch of a nerve will sometimes excite an irritation at the periphery of another branch of the same nerve, which is distributed to another part-e. g., a toothache is often accompanied by pain in the ear, both pains being relieved by the in troduction into the auditory canal of a little laudanum or chloroform ; the earache is aggravated, however, by the application of cold to the tooth, and disappears on its extraction. Irritation of the gastric branches of the vagus by improper food will sometimes give rise in children to reflex spasm of the glottis; irritation of the pulmonary branches of the same nerve by enlarged bronchial glands to a violent convulsive cough. We know also that an impression at the peripheral extremity of a sensitive nerve may produce such a change in that part of the nervous centre from which it arises as to excite a motor or sensitive nerve implanted near to it; and that if a sensitive nerve be stimulated at its origin in the brain, a sensation is produced which is referred to its peripheral extremity. If a motor nerve be in this manner excited a reflex action results, amongst the numerous examples of which that of winking may be cited. The afferent nerve is in this case a branch of the superior maxillary division of the fifth cranial nerve, and the efferent, or motor nerve, is the portio dura. The pain over the brow, sometimes induced by the swallowing of ice or cold water, at others by a derangement of stomach digestion, furnishes us with an example of a reflected sensation. The sympathy between the ear and the larynx affords us another in stance. The impression produced by irritation of the auditory canal, in those who are the subjects of this peculiarity, is pro bably conveyed by the auriculo-temporal branch of the in ferior maxillary division of the fifth cranial nerve to the deep origin of its sensitive root, which is in close proximity to the deep origin of the vagus in the floor of the fourth ventricle. Here a change is in all probability effected in the grey matter, which results in the stimulation of the root of the vagus. The irritation is referred to the larynx, because the medulla oblongata is wont to receive impressions from that organ through this nerve. As a consequence, the expiratory muscles necessary for coughing are set in action to free the larynx of the supposed irritation. Sometimes the cough is accompanied by vomitingan action which not only results from irritation of the peripheral extremity of the vagus, as when emetics produce it, but from an excitation of the cranial origin of this nerve. The cough from teething, which it is so important to diagnose from that of croup, bronchitis, \&c., and which usually ceases as soon as the gum lancet has been judicionsly employed, furnishes us with a third example of a reflected sensation. Dentists appear to be well acquainted with the fact that a continued irritation of the dental branches of the fifth cranial nerve will sometimes produce a cough at almost any age, and that in such cases the stump of a tooth is frequently the cause of this annoyance.

With reference to the implantation of the roots of the fifth cranial nerve and the vagus, it may be observed that Stilling, in his work on the Medulla Oblongata and Pons Varolii, describes certain special deposits of grey matter or nuclei in the floor of the fourth ventricle, and states that they are the deep origins of the spinal accessory, vagus, glosso-pharyngeal, and hypoglossal nerves. Engravings are given representing the nuclei of the varus and glosso-pharyngeal in juxtaposition. Langenbeck and Förg hold, however, that the accumulation of grey matter considered by Stilling to be the deep origin of the glosso-pharyngeal nerve is not so, but is in truth the nucleus of origin of the greater or sensitive root of the fifth cranial nerve. Although unanimity of opinion does not yet prevail as to the exact origin of the greater root of this important nerve, it would seem to have been traced beyond a doubt into the floor of the fourth ventricle, from which the vagus also arises.

Reference is made in the quotations given at the commencement of this paper to the occasional occurrence of vomiting when the external auditory meatus is irritated. Although the majority of aurists have omitted in their works any mention of the intimate sympathy between the ear and the stomach, yet cases very frequently arise where a derangement of the digestive organs is the sole cause of deafness. Yearsley, however, in his "Deafness Practically Illustrated," treats of "stomach deafness;" and justly wonders at the omission, especially as the sympathy between the eye and the stomach has been long recognised in the occasional production of amau- 
rosis by dyspeptic disorders. Arnold furnishes us with an interesting example of this sympathy between the ear and the stomach in the case of a child who suffered from chronic vomiting which defied for a long time all curative measures, but was immediately removed on the extraction from each ear of $a$ bean which had been introduced in play.

There is on record a case of dysphonia caused by spasm of the left true vocal cord, and accompanied by pain in the left par. This spasm was considered to depend, in all probability, on the pressure of the left vagus, where the recurrent laryngeal branch is given off, by an aneurism; but several examinations failed in eliciting any proof of the existence of a tumour. The patient complained bitterly of the pain in the ear, and stated that it was "a piercing pain right inside"-endeavouring to enforce his remark by inserting his finger in the meatus. No allusion is made by the reporter of the case to the condition of the auditory canal, which was probably not examined.

Dr. Gull states that pain in the ear is not uncommon in connexion with thoracic aneurisms. My own experience, however, does not support this opinion; for only once have I encountered aural pain in connexion with that disease. Although the presence of a tumour was not demonstrable, yet all the symptoms undoubtedly pointed to the existence of some body pressing on the left recurrent laryngeal nerve in its thoracic course. The irritation by the same tumour of one or more of the numerous pulmonary or cardiac branches of the vagus in such close vicinity would readily account for the pain in the ear. The explanation already given of the sympathy between the ear and the larynx applies in this case, if the direction from which the impression starts be reversed.

In conclusion, the foregoing observations may thus be summed up :-

1. The sympathy between the ear and the larynx, as well as the stomach, has been long known, although the majority of recent writers seem to have overlooked it.

2. This sympathy is not manifested in every individual, but in about seventeen per cent., and seems to depend on a state of hyperæsthesia of the nerve which supplies the auditory canal.

3. The nerve of the ear concerned in the production of this phenomenon cannot be a branch of the vagus, as Romberg and Toynbee have affirmed, but is in all probability a branch of the fifth cranial nerve.

4. This sympathy is an example of a reflected or sympathetic sensation, in which the connexion between the nerves concerned takes place in the nervous centre.

5. Cases occasionally occur where a cough is solely dependent on the existence of some source of irritation in the auditory canal.

6. The explanation of the sympathy between the ear and the larynx enables us to understand the mode in which pain of the ear becomes occasionally a symptom of a thoracic aneurism.

One of my chief objects in bringing before the notice of my professional brethren this sympathetic connexion is to introduce to them what may be called an ear-cough, and to strongly advise them to examine the auditory canals in all cases of obstinate cough, where none of the more frequent causes of this symptom can be discovered.

Scarborough, $\Lambda$ l)il, 1866

\section{ON GENERAL DIPHTHERIA.}

BY JAMES FOWLER, EsQ., M.R.C.S.,

HON. SURQEON TO CLAYTON'S HOSPITAI AND DISPENSARY, WATRTIKLD.

IN addition to an unusual number of cases of scarlatina and erythematous sore-throat in this district, there have lately been several of true diphtheria. At the beginning and height of the epidemic, though there was considerable prostration, the local symptoms were most characteristic - limited in a general way to the fauces, and accompanied by well-marked fibrinous exudation, slowly thrown off, and re-formed many times, perhaps, before the issue of the case either in death or recovery. Latterly, however, such isolated manifestations have been less frequently observed. Instead, alarming general depression has been amompanied by a peculiar kind of inflammation, varying much in its intensity, in the organ which it primarily affects, and in the amount of fibrin which it deposits; but constant in the suddenness of its appearance, the insidi- ousness and ungovernableness of its progress, and the speedy fatality of its termination. Good illustrations of both forms occurred in an outlying, ill-drained village, built partly round a large sheet of almost stagnant water, strongly impregnated with decaying vegetable matter; bronchocele being so common here that it may almost be said to be endemic. It must be added, however, that scarcely less serious instances have been noticed in the town itself, and in situations where not only the circumstances and occupations of patients, but the drainage, ventilation, and construction of their homes, were everything that could be wished. The following is selected, not only on account of the infrequency of such cases, and the still greater scarcity of adequate records of them, but because there are several accidental circumstances connected with it which add materially to its interest.

Mary $\mathrm{A}-$, agel thirty-nine, of temperate and regular habits, at home with a married sister in one of the healthiest parts of the town, without having been at all exposed to cold or wet, went to bed on March 10th complaming of a little sore-throat, with hoarseness and difficnlty of swallowing. Of this, however, thinking it proceeded from cold, she took no notice until towards morning, when it became much worse, and increased until about eight A.M. on the $20 \mathrm{th}$, when I was sent for, and found her propped up in bed, breathing noisily and with much difficulty. There neither was nor had been any cough or expectoration of any kind, but she was very anxious and prostrate, and spoke huskily or in a whisper, evidently with much pain and effort. Placing her finger on the pomum Adami as the seat of chief suffering, she said, "It is all here-choking." The mouth could not be got sufficiently wide open for the throat to be properly examined, the parotid and submaxillary glands on the right side being swollen and tender; but there was evidently a good deal of diffused redness in the fauces, especially about the tonsils. There did not appear to be any mischief in the chest; but it was difficult to speak accurately for this, as loud croupy laryngeal breathing quite hid all finer sounds in whatever part of the chest the stethoscope was placed.

Under the impression that I had got to deal with a case of acute laryngitis, I simultaneously fomented the throat with hot water and sponges, applied blistering liquid to the back of the neck, and gave forty grains of powdered ipecacuanha in water. The dose was swallowed only with much pain and difficulty, and, though repeated in ten minutes, produced neither the slightest attempt at vomiting nor the least improvement in the breathing. The latter was, in fact, so much worse at the end of half an hour, the pulse so rapid and yet so faint and irregular, the countenance so pale and dusky, that suffocation seemed imminent, and laryngotomy was accordingly performed. There was nothing remarkable in the operation; only slight bleeding occurred, and that ceased the moment the larynx was opened. The relief obtained, though very considerable, was not perfectly satisfactory. The breathing was at once made quite easy, and, as a large canula was used, almost noiseless; the pulse, though still very soft, became regular, fuller, and less frequent; but the countenance had a cadaverous expression, and sallowness and anxiety marked on it, for which there was no adequate explanation, especially as the chest was found (and it could now be examined accurately) perfectly healtby. Four hours afterwards, in order to obtain efficient nursing and attendance, she was removed in a close cab to the hospital - a distance of only a few hundred yards ; and wrapped in blankets, with a couple of large sponges previously immersed in boiling water applied to the throat, every possibility of a chill being taken was avoided; the skin was, indeed, very soon after, quite moist and perspiring. There being considerable inflammation of the tonsils, besides external swelling and infiltration of the subcutaneous areolar tissue in front of the neck, with swelling and tenderness of the lymphatic glands on both sides, six leeches were applied, which gave immediate relief, at least so far as the pain was concerned. But before evening it was evident that a smart attack of bronchitis was impending, loud rhonchi being audible all over the chest. The room was accordingly kept uniformly at $60^{\circ} \mathrm{F}$., and the air saturated with watery vapour, at the same time that diaphoretic doses of antimony were given, and the bowels cleared by calomel and salts.

The night was passed restlessly, and a careful examination. in the morning (March 2lst) showed that the inflammation had spread to the smaller tubes throughout the chest; the larger ones by this time containing viscid mucus, which was expectorated with great difficulty. The back of the left lung, besides, was dnll; but all fine anscultation was out of the question, loud rattles, and growling, groaning, and snoring 